\title{
Dynamical Stability of an Ion in a Linear Trap as a Solid-State Problem of Electron Localization
}

\author{
G.P. Berman ${ }^{1}$, A.R. Bishop ${ }^{1}$, D.F.V. James ${ }^{1}$, R.J. Hughes ${ }^{2}$, and D.I. Kamenevev ${ }^{1,3}$ \\ 1 Theoretical Division, Los Alamos National Laboratory, Los Alamos NM 87545 \\ 2 Physics Division P-23, MS H-803, Los Alamos National Laboratory, Los Alamos NM 87545 \\ ${ }^{3}$ Center for Nonlinear Studies, MS B-258, Los Alamos National Laboratory, \\ Los Alamos NM 87545
}

\begin{abstract}
When an ion confined in a linear ion trap interacts with a coherent laser field, the internal degrees of freedom, related to the electron transitions, couple to the vibrational degree of freedom of the ion. As a result of this interaction, quantum dynamics of the vibrational degree of freedom becomes complicated, and in some ranges of parameters even chaotic. We analyze the vibrational ion dynamics using a formal analogy with the solid-state problem of electron localization. In particular, we show how the resonant approximation used in analysis of the ion dynamics, leads to a transition from a two-dimensional (2D) to a one-dimensional problem (1D) of electron localization. The localization length in the solid-state problem is estimated in cases of weak and strong interaction between the cites of the $2 \mathrm{D}$ cell by using the methods of resonance perturbation theory, common in analysis of $1 \mathrm{D}$ time-dependent dynamical systems.
\end{abstract}

PACS numbers: 32.80.Pj, 42.50.Vk, 05.45.Mt 


\section{INTRODUCTION}

The problem of quantum dynamics for Hamiltonian systems with time-periodic perturbation (TPS) can be formulated in terms of an equivalent solid-state problem of electron localization on a lattice. Such kinds of connections were discussed for different models int, 目 (see also references therein). However, most results are obtained for quantum kicked systems, such as a quantum kicked rotor or a quantum kicked oscillator. These systems are convenient for both analytical and numerical analysis because instead of differential equations one can use discrete quantum maps. At the same time, the existence of periodic kicks suggests that the external field involves an infinite number of harmonics with equal amplitudes. In more common physical situations, there are only few harmonics in the perturbation. In particular, such a situation occurs when an ion trapped in a linear ion trap interacts with two laser field with close frequencies 3 . In this case, the internal degree of freedom of the ion (related to the electron dynamics) interacts with the vibrational degree of freedom. This interaction can result in complicated and even chaotic dynamics of the vibrational degree of freedom of the ion. The analysis of the stability of the ion in this system can be performed using a model of a quantum harmonic oscillator perturbed by a monochromatic wave (MPO) .

In this paper we show that the problem of stability of a MPO can be formulated in terms of localization of an electron in a 2D solid-state system (SSS). The resonance approximation, common in treatment of TPSs, is used to reduce the effective dimensionality of the SSS in the case of relatively small interaction of the ion with the laser field. In order to compare two completely different systems, a similarity in the formal description of the TPS and a spaceperiodic SSS is exploited. Namely, in the TPS we use the time-periodicity of the perturbation and employ a Floquet formalism, while in the SSS we exploit a space-periodicity and use the Bloch theorem.

The paper is organized as follows. In Section I we describe the MPO model of an ion trapped in a linear ion trap and interacting with two laser fields with close frequencies. In Section II, we discuss the general procedure which allows to treat an 1D TPS on the same 
ground as a 2D SSS. In the case of a small perturbation, the resonance approximation is used in Section III to decrease the effective dimensionality of the SSS from two to one. The localization length in the SSS is estimated in Section IV by calculating the size of the chaotic region in the corresponding TPS, in the situation when the interaction between the sites of 2D SSS is strong. Concluding remarks are given in Conclusion.

\section{THE VIBRATIONAL HAMILTONIAN}

In the following 3 , we assume that two laser beams, designated the pump (p) and the Stokes (s), with slightly different frequencies, $\omega_{p}$ and $\omega_{s}$, respectively, interact with the ion trapped in a linear ion trap. Both beams are assumed to be plane polarized in the z-direction with the amplitudes of the electric field, $\mathcal{E}_{z}^{(p)}$ and $\mathcal{E}_{z}^{(s)}$, and the wave vectors, $\mathbf{k}_{p}$ and $\mathbf{k}_{s}$. The Hamiltonian, including the effect of the harmonic evolution of the ion along the weak axis of the trap (but excluding the internal free evolution) is,

$$
\hat{\mathcal{H}}=\frac{\hat{p}^{2}}{2 M}+\frac{M \omega^{2} \hat{x}^{2}}{2}+\frac{\varepsilon}{k} \cos (k \hat{x}-\Omega t),
$$

where where $\hat{p}$ and $\hat{x}$ are the $x$-components of the momentum and the coordinate of the ion, $t$ is the time, $M$ is the mass of the ion, $\omega$ is the frequency of the ion vibrations in the linear $\operatorname{trap}, \varepsilon=2 \chi k\left|\mathcal{E}_{z}^{(p)} \mathcal{E}_{z}^{(s) *}\right|, \Omega=\omega_{p}-\omega_{s}, k=\left(\mathbf{k}_{p}-\mathbf{k}_{s}\right) \cdot \mathbf{e}_{\mathbf{x}}$, $\mathbf{e}_{\mathbf{x}}$ is a unit vector in $x$-direction, $\chi=A \pi \epsilon_{0} / 4 \nu^{3} \Delta(\nu$ and $A$ being, respectively, the wavenumber and the Einstein A coefficient for the transition between the upper and lower manifolds, $\Delta$ is the laser detuning and $\epsilon_{0}$ the permitivity of free space).

In the dimensionless form the Hamiltonian (1) reads,

$$
\hat{H}=\frac{\hat{\mathcal{H}}}{\left(M \omega^{2} / k^{2}\right)}=-\frac{h^{2}}{2} \frac{\partial^{2}}{\partial X^{2}}+\frac{X^{2}}{2}+\epsilon \cos (X-\mu \tau)=\hat{H}_{0}+V(X, \tau),
$$

where $\hat{H}_{0}$ is the Hamiltonian of a linear oscillator,

$$
X=k x, \quad \tau=\omega t, \quad \epsilon=\frac{\varepsilon k}{M \omega^{2}}, \quad h=\frac{\hbar k^{2}}{M \omega}, \quad \mu=\frac{\Omega}{\omega}=N+\delta .
$$


Here $h$ is a dimensionless Planck constant, $N$ is the (positive integer) resonance number, and $\delta$ is the detuning from the resonance.

The classical analog of the Hamiltonian (2) is,

$$
H=\frac{X^{2}}{2}+\frac{P^{2}}{2}+\epsilon \cos (X-\mu \tau)
$$

where $P=k p / M \omega$ is the dimensionless momentum.

In the action-angle variables, $(I, \vartheta)$, the classical Hamiltonian (甘) takes the form,

$$
H=I+\epsilon \cos [k r(I) \sin \vartheta-\mu \tau]
$$

where $X=k r(I) \sin \vartheta, P=k r(I) \cos \vartheta, k r=\sqrt{X^{2}+P^{2}}=\sqrt{2 I}$ is the dimensionless amplitude of oscillations, $I$ is the dimensionless action, measured in units of $I_{0}=M \omega / k^{2}$, and $\vartheta$ is the phase of oscillations.

\section{CONNECTION WITH A 2D SOLID-STATE LOCALIZATION PROBLEM}

We write the solution to the Schrödinger equation,

$$
i h \frac{\partial \Psi(X, \tau)}{\partial \tau}=\hat{H} \Psi(X, \tau)
$$

in the form of series over the eigenfunctions, $|n\rangle \equiv \phi_{n}(X)$, of the harmonic oscillator Hamiltonian, $\hat{H}_{0}$,

$$
\Psi(X, \tau)=\sum_{n=0}^{\infty} c_{n}(\tau)|n\rangle .
$$

Then we obtain the equations for the complex amplitudes, $c_{n}(\tau)$,

$$
\begin{gathered}
i h \frac{d c_{m}(\tau)}{d \tau}=h(m+1 / 2) c_{m}(\tau)+\epsilon \sum_{n=-m}^{\infty}\langle m|\cos (X-\mu \tau)| m+n\rangle c_{m+n}(\tau)= \\
h(m+1 / 2) c_{m}(\tau)+\frac{\epsilon}{2} \sum_{n=-m}^{\infty}\left(e^{-i \mu \tau} F_{m, m+n}+e^{i \mu \tau} F_{m, m+n}^{*}\right) c_{m+n}(\tau) .
\end{gathered}
$$

In Eq. (8), $F_{m, m+n}$ is the matrix element 1 ,

$$
F_{m, m+n}=\left\langle m\left|e^{i X}\right| m+n\right\rangle=\frac{i^{n} h^{n / 2} e^{-h / 4}}{2^{n / 2} \sqrt{(m+1)(m+2) \ldots(m+n)}} L_{m}^{n}\left(\frac{h}{2}\right),
$$


where $L_{m}^{n}$ is the Laguerre polynomial. When $m \gg 1$, the Laguerre polynomials can be expressed in terms of the Bessel functions, $J_{n}$ 日, as

$$
L_{m}^{n}\left(\frac{h}{2}\right)=\left(\frac{2 m}{h}\right)^{n / 2} J_{n}(\sqrt{2 m h}),
$$

where the argument of the Bessel function, $\sqrt{2 m h}=k r_{m}$, is the quantized dimensionless amplitude of oscillations of the harmonic oscillator. Using Eqs. (9) and (10) the matrix elements can be written in the form,

$$
F_{m, m+n}=\frac{i^{n} m^{n / 2} e^{-\frac{h}{4}}}{\sqrt{(m+1) \ldots(m+n)}} J_{n}(\sqrt{2 m h}) .
$$

Since the Hamiltonian (2) is periodic in time the solution of the Schrödinger equation (8) can be written as,

$$
c_{m}^{q}(\tau)=e^{-i \sigma_{q} \tau / h} A_{m}^{q}(\tau)
$$

where $\sigma_{q}$ is a quasienergy, measured in units of $M \omega^{2} / k^{2}, c_{m}^{q}(\tau)$ is the quasienergy (QE) function, and $A_{m}^{q}(\tau)$ is a periodic function with the period, $T=2 \pi / \mu$,

$$
A_{m}^{q}(\tau+2 \pi / \mu)=A_{m}^{q}(\tau)
$$

The quasienergy functions are the eigenfunctions of the evolution operator, $\hat{U}$, for one period, $T$, of the external field,

$$
\hat{U}(T) c_{m}^{q}(\tau)=e^{-i \sigma_{q} T / h} c_{m}^{q}(\tau)
$$

The evolution operator for one period of the external field, $\hat{U}(T)$ is,

$$
\hat{U}(T)=\hat{T} e^{-i \int_{0}^{T} \hat{H}(\tau) d \tau},
$$

where $\hat{T}$ is the ordering operator, $\hat{H}(\tau)$ in our problem is given by Eq. (2). In our numerical calculations, presented below, we consider only the QE states at the time $\tau=0$, so that $c_{m}^{q}(0)=A_{m}^{q}(0) \equiv A_{m}^{q}$ 円

\footnotetext{
${ }^{1}$ If the spectrum, $\sigma_{q}$, and $\mathrm{QE}$ functions, $A_{m}^{q}$, are known, one can trace evolution of the quantum system at the times $\tau_{s}=s T$, where $s=0,1,2 \ldots$ (see, for example ${ }^{6}$ ).
} 

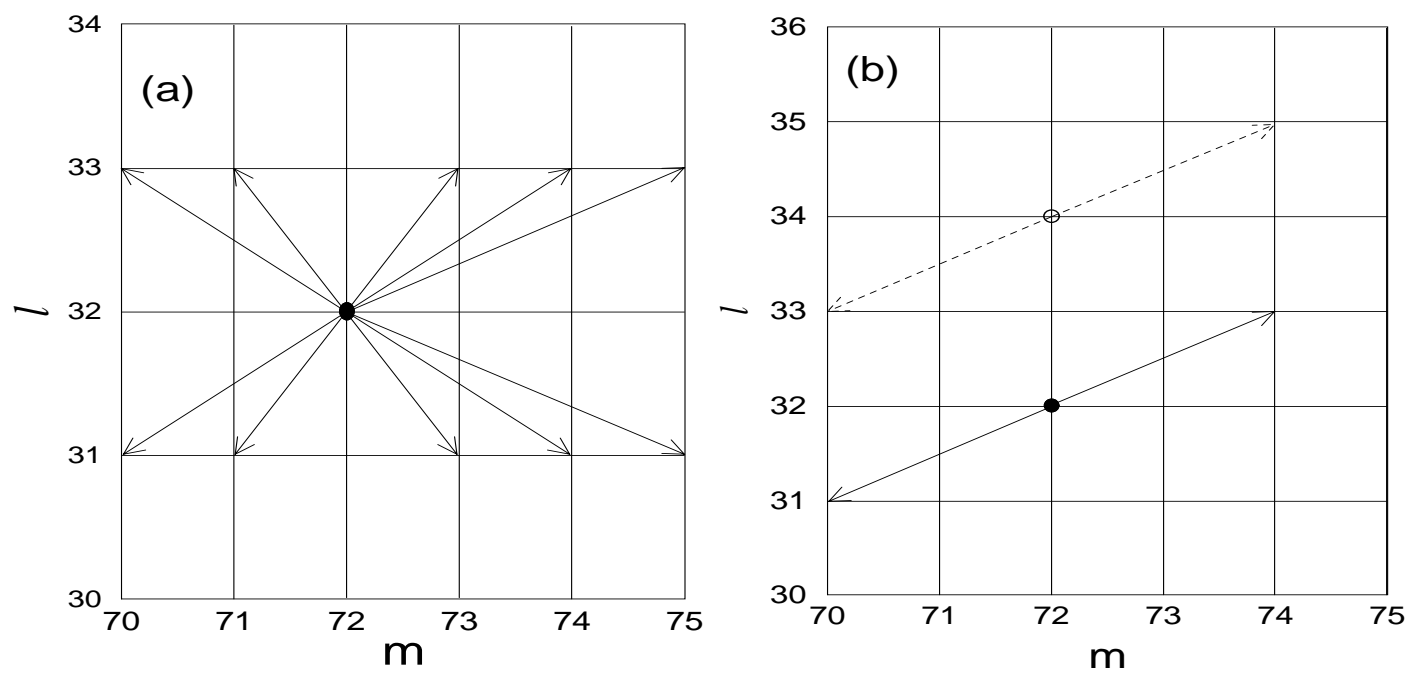

FIG. 1. (a) Some possible transitions on a 2D lattice given by Eq. (18). (b) Possible transitions on a $2 \mathrm{D}$ lattice at small $\epsilon$, given by Eq. (24). $N=2$.

Substitution (12) in (8) gives the equations for $A_{m}^{q}$,

$$
\begin{gathered}
\sigma_{q} A_{m}^{q}(\varphi)=-i h \mu \frac{d A_{m}^{q}(\varphi)}{d \varphi}+h(m+1 / 2) A_{m}^{q}(\varphi)+ \\
\frac{\epsilon}{2} \sum_{n=-m}^{\infty}\left(e^{-i \varphi} F_{m, m+n}+e^{i \varphi} F_{m, m+n}^{*}\right) A_{m+n}^{q}(\varphi), \quad \varphi \equiv \mu \tau .
\end{gathered}
$$

Expanding the function $A_{m}^{q}(\varphi)$ in a Fourier series,

$$
A_{m}^{q}(\varphi)=\sum_{l=-\infty}^{\infty} A_{m, l}^{q} e^{-i l \varphi}
$$

we derive the following equation for the amplitudes, $A_{m, l}^{q}$,

$$
E_{q} A_{m, l}^{q}=h(m-\mu l) A_{m, l}^{q}+\frac{\epsilon}{2} \sum_{n=-m}^{\infty}\left(F_{m, m+n} A_{m+n, l+1}^{q}+F_{m, m+n}^{*} A_{m+n, l-1}^{q}\right),
$$

where $E_{q}=\sigma_{q}-h / 2$. Equations (18) can be interpreted as a problem for electron localization on a $2 \mathrm{D}$ lattice. Indeed, one can consider the complex coefficients, $A_{m, l}^{q}$, as the complex amplitudes of probability to find an electron on a $2 \mathrm{D}$ lattice at the site $(m, l)$, where $0 \leq$ $m<\infty,-\infty<l<\infty$. Some possible transitions in the system (18) are shown in Fig. 1 (a). The particle, initially located at the site with the indexes $\left(m_{0}, l_{0}\right)$ can jump at the sites $\left(m_{0} \pm n, l_{0} \pm 1\right)$, where $n$ is an integer number. 


\section{THE RESONANCE APPROXIMATION}

When the interaction amplitude is small, $\epsilon \ll 1$, the 2D SSS described by Eq. (18) can be reduced to the $1 \mathrm{D}$ SSS described by the equation with only one index. We divide both parts of Eq. (18) on $\mu$ and, taking into account that $\delta \ll N$ and $1 / \mu \approx 1 / N-\delta / N^{2}$, obtain

$$
\frac{E_{q}}{\mu} A_{m, l}^{q}=h\left(\frac{m}{N}-\frac{\delta m}{N^{2}}-l\right) A_{m, l}^{q}+\frac{\epsilon}{2 \mu} \sum_{n=-m}^{\infty}\left(F_{m, m+n} A_{m+n, l+1}^{q}+F_{m, m+n}^{*} A_{m+n, l-1}^{q}\right) .
$$

We assume that $\delta$ is small, so that $\delta m / N^{2} \ll 1$ for all considered values of $m$, or $\delta=0$. Then, in the zeroth order approximation we have from Eq. (19),

$$
\left(\frac{m}{N}-l\right) A_{m, l}^{q}=\frac{E_{q}^{(0)}}{\mu h} A_{m, l}^{q}
$$

It follows from Eq. (20) that if $A_{m, l}^{q} \neq 0$, than $E_{q}^{(0)} / \mu h=(m / N)-l$. Since the ratio, $E_{q}^{(0)} / \mu h$, is defined by modulus 1 (see Eq. (14)), we can write, $E_{q}^{(0)} / \mu h=0$ \& Then Eq. (20) takes the form,

$$
(m-N l) A_{m, l}^{q}=0
$$

Hence,

$$
\begin{array}{cc}
A_{m, l}^{q}=0 & \text { for } \quad m \neq N l, \\
A_{m, l}^{q} \equiv A_{m}^{q} & \text { for } \quad m=N l .
\end{array}
$$

The next order approximation for $m=N l$ yields,

$$
\left(E_{q}-h \delta m / N\right) A_{m}^{q}=\frac{\epsilon}{2}\left(F_{m, m+N} A_{m+N}^{q}+F_{m, m-N}^{*} A_{m-N}^{q}\right)
$$

\footnotetext{
${ }^{2}$ We assume that $(m / N)-l$ is an integer for some initial state $m_{0}$. If not, one may introduce the quasienergy, $E_{q}^{\prime}=E_{q}-\mu h\left\{m_{0} / N\right\}$ and a new index, $m^{\prime}=m-\left\{m_{0} / N\right\} N$, and solve Eqs. (19) and (20) for $E_{q}^{\prime}$ and $m^{\prime}$ instead of $E_{q}$ and $m$. Here $\{x\}$ is the fractional part of $x$.
} 
where $E_{q}=E_{q}^{(1)}$ (we do not consider the higher order approximations). As one can see from Eq. (21), in the $m$-direction only hops on the distance $N$ are allowed. Thus, the 2D problem, given by Eq. (18), is reduced in the case $\epsilon \ll 1$ to the 1D problem described by Eq. (24).

The localization properties of the quantum states in the resonance approximation, given by Eq. (24), are defined by the structure of the matrix elements, $F_{m, m+N}$. If the matrix elements are periodic functions of $m$, all the eigenstates are extended and the spectrum is continuous. This situation is common for solid-state system 6 . In the system under consideration, the matrix elements (11) are non-periodic. On this reason, as will be shown below (see also Refs. 5 (t) the quantum states are localized and the spectrum is discrete.

The matrix elements given by Eq. (11), oscillate as a function of $m$. At the points, $m_{0}$, where the matrix elements are close to zero,

$$
F_{m_{0}, m_{0}+N} \sim J_{N}\left(\sqrt{2 m_{0} h}\right) \approx 0
$$

the transition probability is very small. As a consequence, such points becomes the dynamical barriers to the probability flow $\mathrm{Z}$, and divide the Hilbert space, labeled by index, $m$, into the relatively independent parts - resonance cells 8 . Most of the eigenstates given by Eq. (24) are concentrated inside these cells. The average localization length, $\left\langle\lambda_{i}\right\rangle$, for the states in the $i$-th cell does not exceed the size of the cell. The cell boundaries are defined by Eq. (25), i.e. $\left\langle\lambda_{i}\right\rangle \leq m_{i+1}-m_{i}$, where $m_{i}$ and $m_{i+1}$ satisfy Eq.(25), so that $\sqrt{2 m_{i} h}$ and $\sqrt{2 m_{i+1} h}$ are, respectively, the $i$-th and $i+1$-th roots of the Bessel function in (25).

Some characteristic QE functions given by Eq. (24) are illustrated in Figs. 2 (b) - (e), for small $\epsilon$. The boundaries of the resonance cells are marked by arrows. One can see from Figs. 2 (b) - (e), that the eigenfunctions are localized inside the cells, but, on the other hand, each eigenfunction is delocalized over $m$ inside a single cell. For example, for the initial states in Fig. 1 (b) with $m=72$ the transitions will occur in the region $66<m<176$ (inside the first cell in Fig. 2 (a)). Note, that for small values of $\epsilon$ and when $\delta=0$, the localization properties of our system are independent of $\epsilon$. This means that an arbitrary small 
perturbation, $\epsilon$, initiates transitions between the sites on the effective rectangular lattice. For small values of $\epsilon$, these transitions take place on 1D sub-lattices shown in Fig. 1 (b).

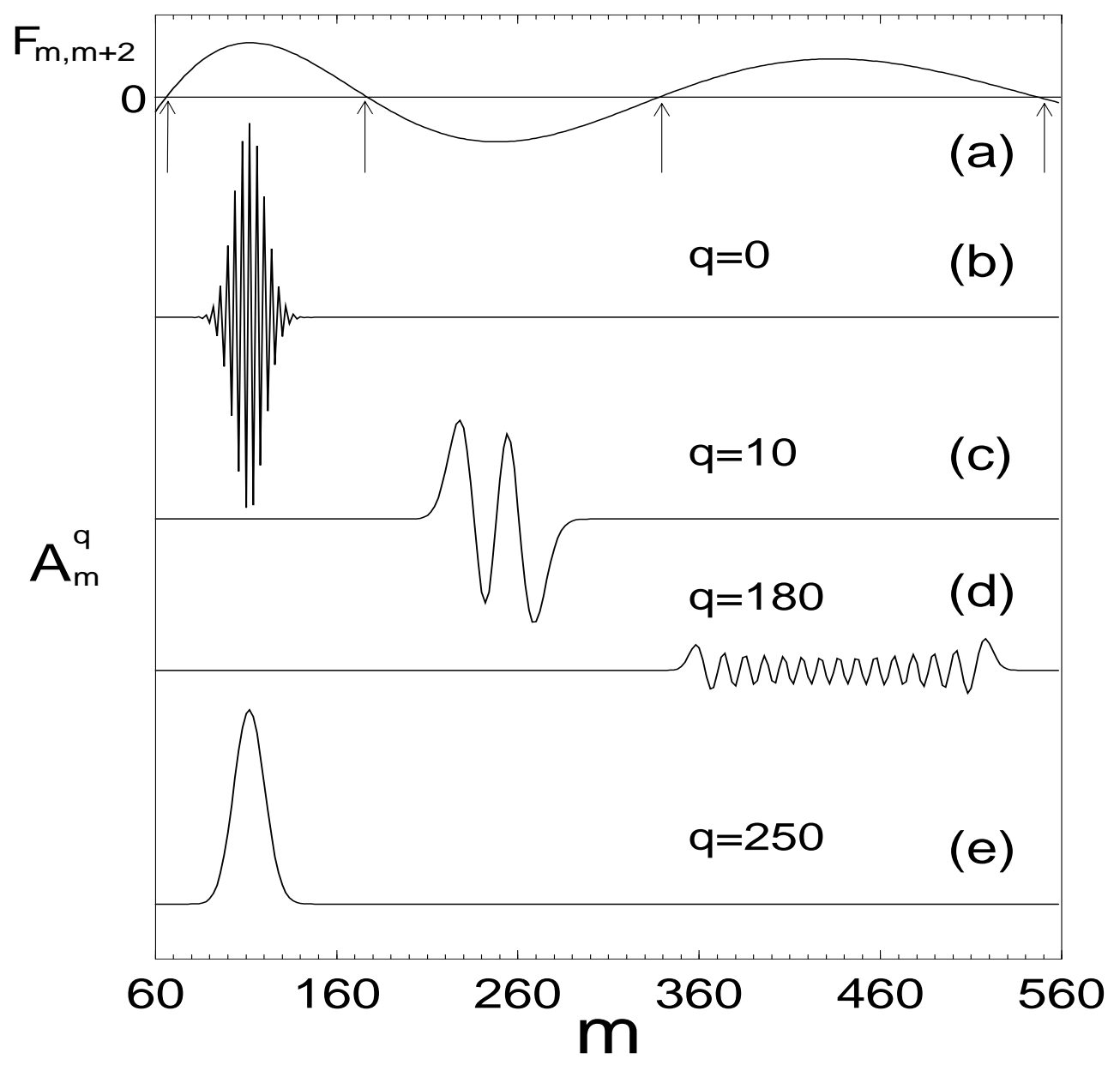

FIG. 2. (a) The matrix elements (in arbitrary units), $F_{m, m+2}$, and (b)-(e) some characteristic QE functions given by Eq. (24) with $h=0.2, \epsilon=0.02, \delta=0, N=2$. Only even values of $m$ are included.

Except for the localized (in the resonance cells) eigenfunctions there exists few eigenfunctions which are delocalized over several resonant cells (DF). One of these representative eigenfunctions is shown in Fig. 3. The DFs have maxima in the regions near the boundaries of the cells marked in Fig. 3 by arrows. Thus, if the initial state is located near the boundary of a resonance cell, say, at $m=66$ in Fig. 3 (in the region near the first arrow), then this state will propagate at a large distance in $m$, over the 1D sub-lattice. This distance can be much larger than the size of the single resonance cell 5 . 


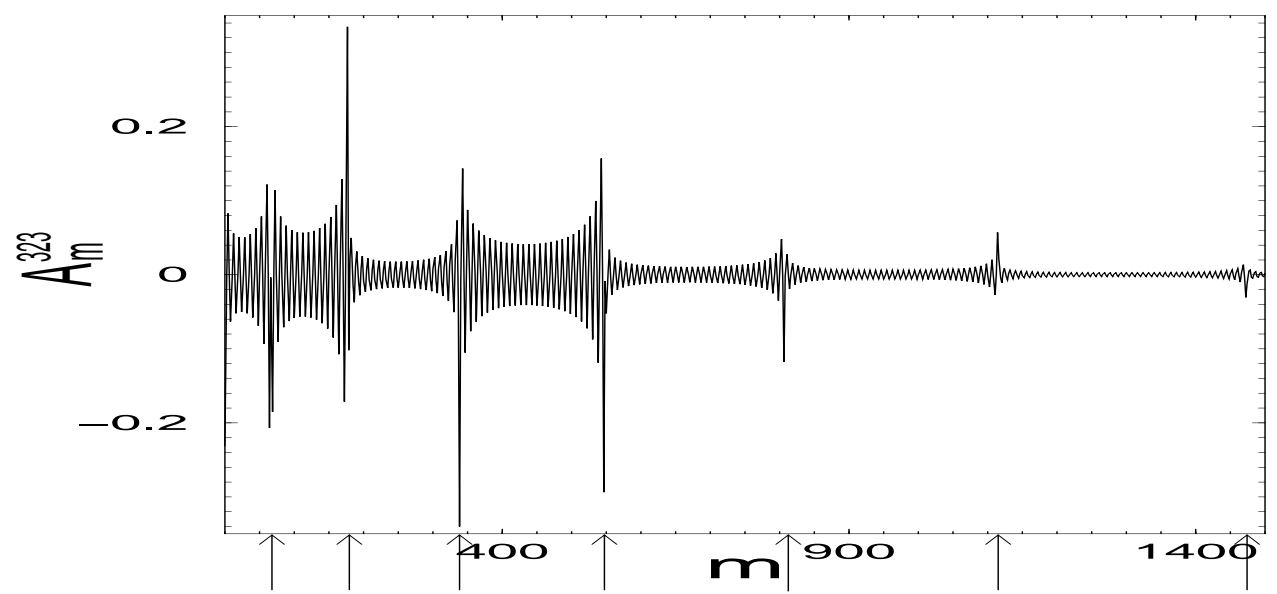

FIG. 3. The characteristic DF (at even $m$ ), $q=323, N=2, h=0.2, \epsilon=0.02, \delta=0$.
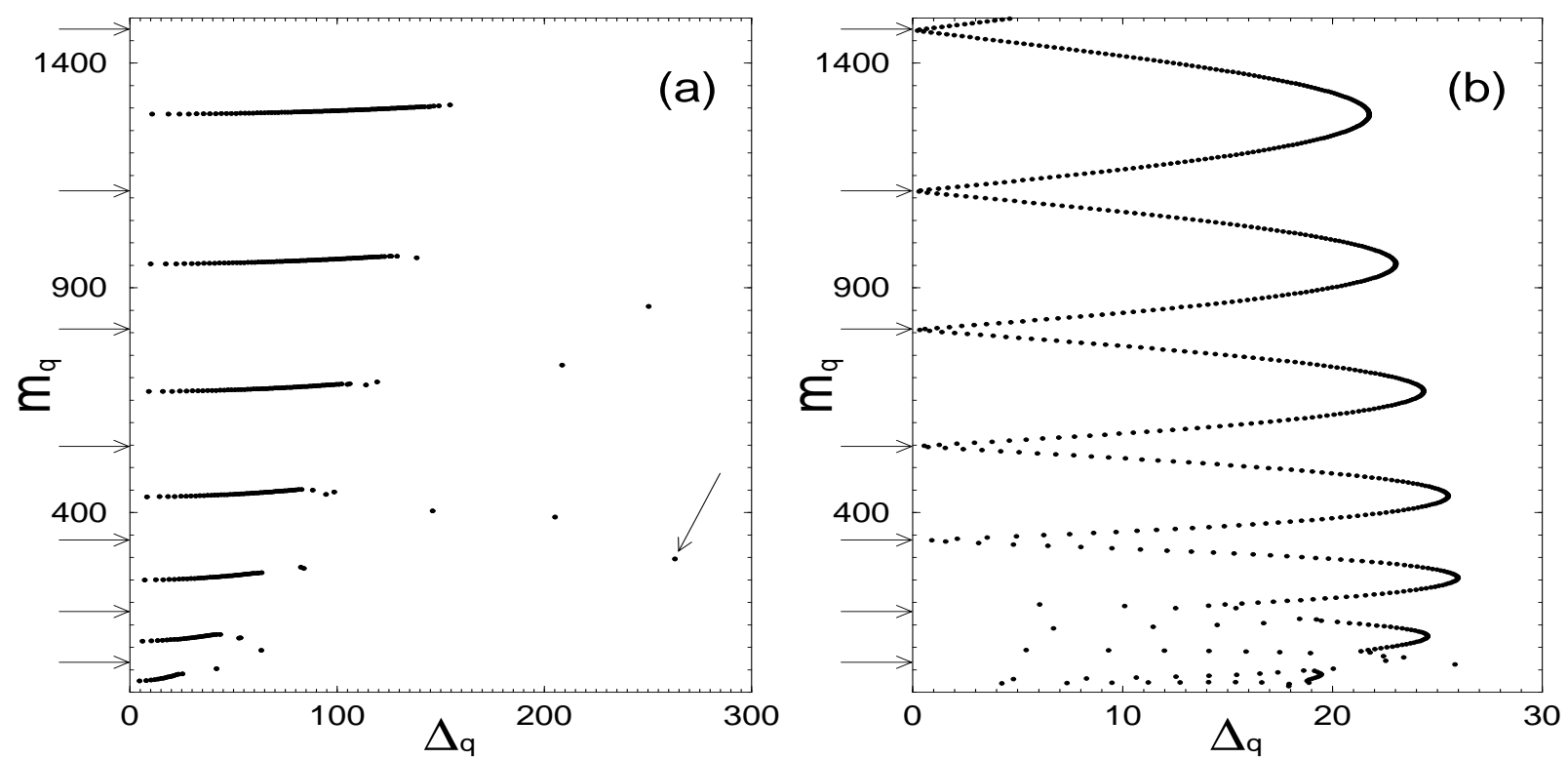

FIG. 4. The plot $m_{q}$ versus $\Delta_{q}$ for $N=2, h=0.2, \epsilon=0.02$ and (a) $\delta=0$, (b) $\delta=0.001$. The boundaries of the resonance cells are marked by the arrows on the $m_{q}$-axis.

The structure of the eigenfunctions can be better understood from the plot of the mean, $m_{q}=\sum_{m}\left|A_{m}^{q}\right|^{2} m$, versus variance, $\Delta_{q}=\left[\sum_{m}\left|A_{m}^{q}\right|^{2}\left(m-m_{q}\right)^{2}\right]^{1 / 2}$, presented in Fig. 4 (a). Each eigenfunction, $A_{m}^{q}$, is represented by one point in the figure. One can see that most of the eigenfunctions are localized inside the resonance cells since their means are located inside the cells and their variances do not exceed the size of the cell. Each row on the figure is formed by the eigenfunctions of one cell. If the initial state is located inside the resonance 
cell, the eigenfunctions of this particular cell define the quantum dynamics. These states make the quantum dynamics localized inside the cell and, at the same time, delocalized over the states, $m$, inside the cellt. In the corresponding solid-state model (24), the localization length at small $\epsilon$ can be identified with the size of the resonance cell.

The DFs are represented in Fig. 4 (a) by the scattered points with large variances. One of the DFs, marked in Fig. 4 (a) by an arrow, is shown in Fig. 3. The DFs can not be attributed to a definite resonance cell since their variances are larger than the size of single cells. As a consequence, the DFs cause delocalization of the states initially concentrated near the boundaries of the cells. However, as shown in Ref.l, the localization length remain finite, because the matrix elements (11) are non-periodic and their amplitudes decrease with $m$ increasing (as $m^{-1 / 4}$ at $m \gg 1$ ).

In the case when the detuning from the resonance (see $(3))$ is not equal to zero $(\delta \neq 0)$, the character of localization depends on the position of an initial state, $m_{0}$ (see Fig. 4 (b)). In the region $m_{0} \gg m_{\max }=\epsilon N / h \delta$ all the states remain exponentially localized in $m$, since in this case Eq. (24) has the solution,

$$
E_{q}=(h \delta m / N) \delta_{m, q}, \quad A_{m}^{q}=\delta_{m, q}
$$

If $m_{0} \ll m_{\max }$ the above discussed effect of localization over the resonance cells takes place. In the intermediate case, when $m_{0} \geq m_{\max }$, the character of localization depends on the position of the state $m_{0}$ inside the resonance cell. (For the parameters in Fig. 4 (b) $m_{\max }=200$.) If $m_{0}$ is located near the boundary of the resonance cell where the condition (25) is satisfied, Eq. (24) has the localized solution (26). Most delocalized functions have their mean, $m_{q}$, at the center of a resonance cell.

As follows from Fig. 4 (b), in the region $m_{0} \geq m_{\max }$ the DFs are absent, since the variance of each function is much less than the size of the cell, whose boundaries are marked in Fig. 4 (b) by arrows. Moreover, the variances of the eigenstates in the near-resonance case in Fig. 4 (b) are substantially smaller than the variances in the exact resonance case shown in Fig. 4 (a). Hence, at small $\epsilon$, the increase of the value of the detuning, $\delta$, always 
leads to localization of the quantum states in the discussed model.

Most of the localization properties of the eigenfunctions, given by Eq. (24), are the quantum manifestation of the classical behavior in the phase space. The classical phase space in the variables $(k r(I), \theta))$, where $\theta=N \vartheta$, $\bmod 2 \pi$, generated by the exact classical Hamiltonian (5), is shown in Fig. 5 (a) for the exact resonance case $(\delta=0)$ and in Fig. 5 (b) for the near resonance case $(\delta=0.001)$.
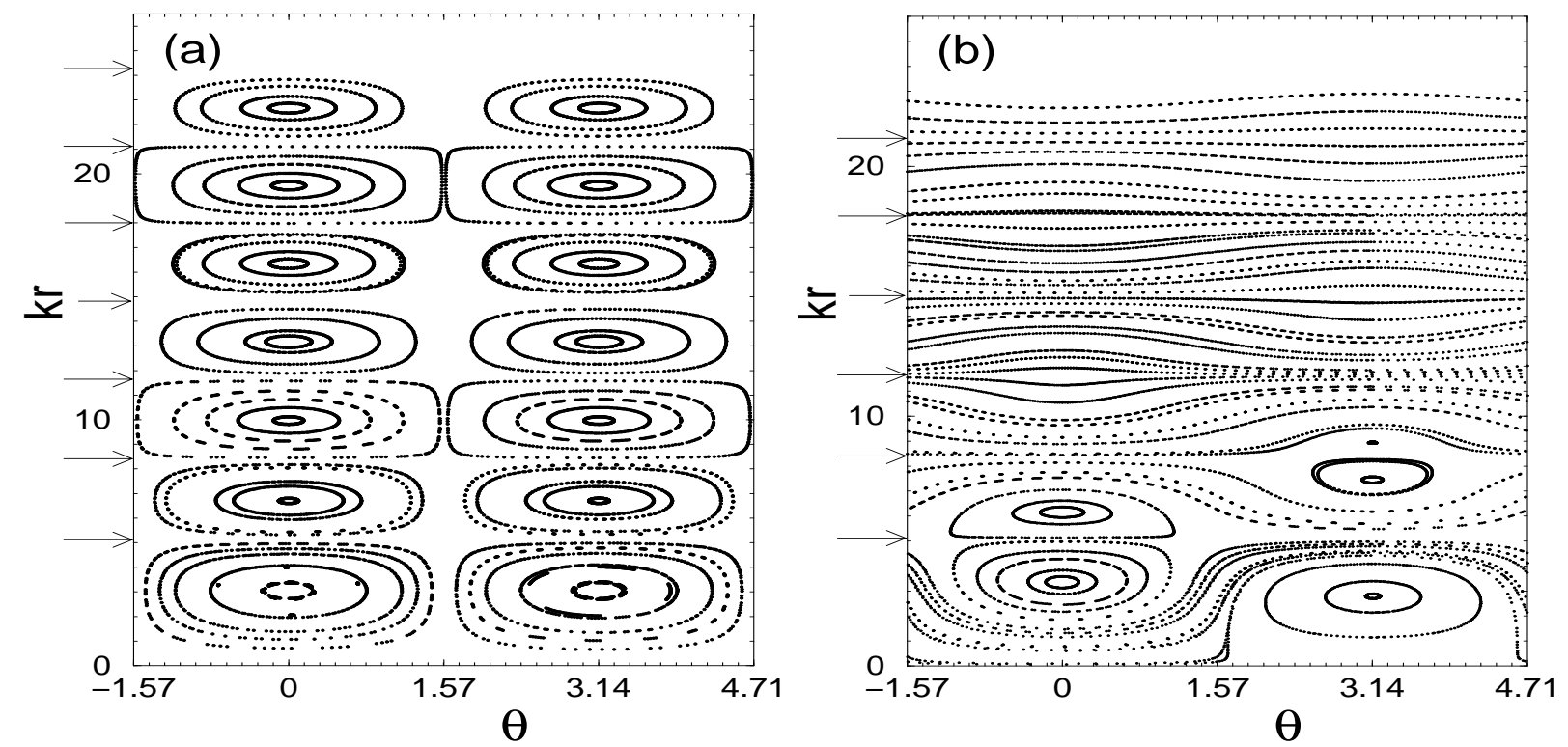

FIG. 5. The classical phase space for (a) $\delta=0$, (b) $\delta=0.001 ; N=2, \epsilon=0.02$. The boundaries of the resonance cells are marked by arrows.

As one can see from Fig. 5 (a), in the case $\delta=0$ the classical phase space is divided into the resonance cells. (Fig. 5 (a) shows only the first seven cells.) The boundaries of the cells, $k r\left(I_{i}\right)=\sqrt{2 I_{i}}$, are marked in Figs. 5 (a), (b) by arrows. As shown in Refs. in the quasiclassical limit the $i$-th boundary of the classical cell, $k r_{i}$, in Figs. 5 (a), (b) corresponds to $i$-th boundary of the quantum cell, $m_{i}$, in $m_{q}$ - axis in Figs. 4 (a), (b), so that $k r_{i}=\sqrt{2 I_{i}}=\sqrt{2 h m_{i}}$.

Each row of points in Fig. 4 (a) is formed by the eigenstates responsible for the dynamics in the corresponding quantum cell. From comparison with the classical dynamics in phase space we can now describe the localization properties of the quantum states, discussed 
above. Each value of $m$ in the quantum system corresponds to a quantized classical action, $I_{m}=m h$, or to the quantized dimensionless oscillation amplitude, $k r_{m}=\sqrt{2 m h}$. Each value of action $I_{m}$ (or $\left.k r_{m}\right)$ corresponds to the set of classical trajectories. Moving along some classical trajectory the particle with some initial value of action, $I_{m}$, can accept the other values in the interval $I_{m_{1}}<I_{m}<I_{m_{2}}$. The corresponding eigenstate will be delocalized over the unperturbed states with the numbers $m$ in the interval, $m_{1}<m<m_{2}$. From the form of the trajectories in Fig. 5 (a) one can see that in the case of exact resonance all quantum states of the single quantum cell should be delocalized over the resonance cell, since in the phase space both the extremal values, $I_{m_{1}}$ and $I_{m_{2}}$, which limit the resonance cell, can belong to the same trajectory.

Similar arguments can be used to analyze the quantum-classical correspondence in the near resonance case. As follows from Fig. 5 (b), at $\delta \neq 0$ in the phase space there is only a finite number of resonance cells (two cells in Fig. 5 (b)). Thus, there is a finite number of quantum resonance cells in the Hilbert space in Fig. 4 (b) (the first two cells). In the off-resonant region (3th-7th cells in Fig. $4(\mathrm{~b})$ ) the degree of delocalization of eigenstates depends on the position of the state in the cell destroyed by the finite detuning, $\delta$. In Fig. 5 (b) the least curved trajectories are located near the separatrices, while the the most curved trajectories are located near the centers of the destroyed cells. As a consequence, in the quantum model the eigenfunctions in Fig. 5 (b) have smallest variance in the region near the separatrices and largest variance near the centers of the destroyed cells.

\section{THE LOCALIZATION LENGTH IN THE CASE OF STRONG INTERACTION}

In the previous sections we considered the dynamics only at small perturbation am-

plitude, $\epsilon \ll 1$. At large values of $\epsilon$ the dynamical chaos appears in the classical 10 and quantum5.11 MPO. As will be shown below, all quantum states in the chaotic area are delocalized over the whole chaotic region. The chaotic dynamics in MPO corresponds to hops 
in different directions in the SSS, as shown in Fig. 1. By estimating the size of the chaotic motion in the MPO we will estimate below the localization length in the SSS.

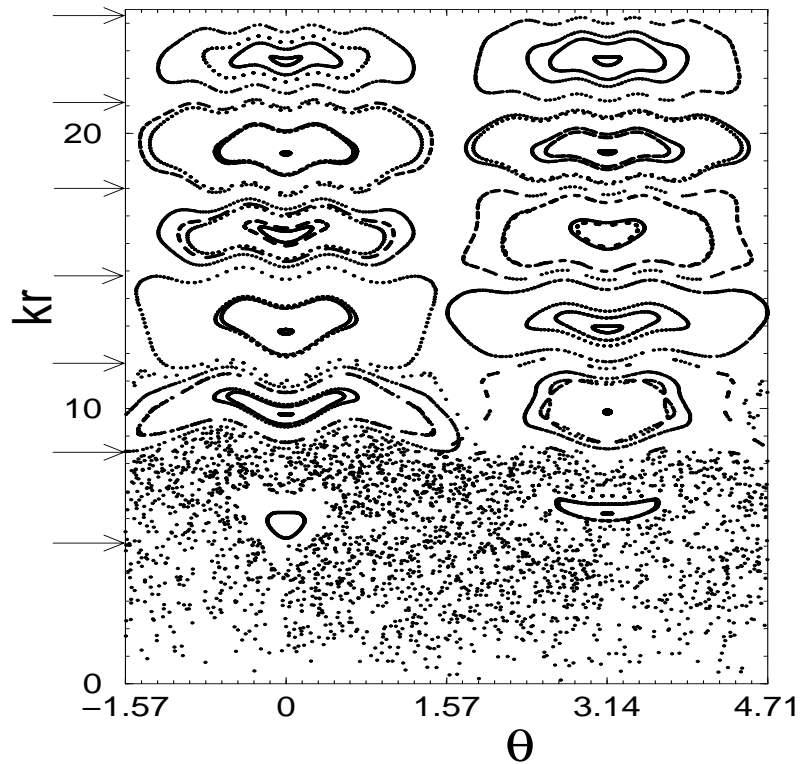

FIG. 6. The classical phase space in the case $\epsilon=3$. Other parameters are: $\delta=0, N=2$. The boundaries of the resonance cells are marked by arrows.
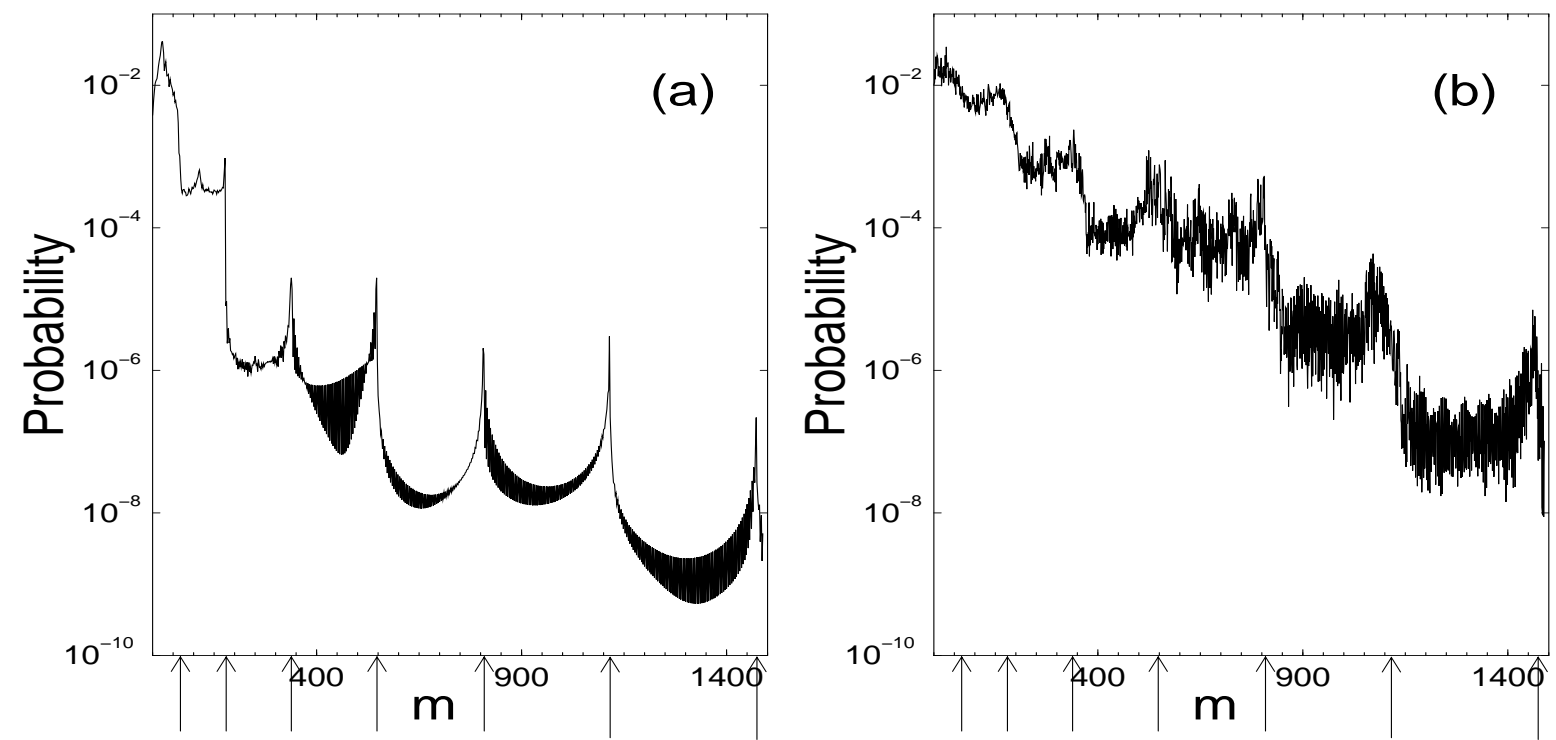

FIG. 7. The time-averaged probability distribution. (a) $\epsilon=0.02$, the averaging has been performed over 100 realizations in the time-interval $\tau=5000-105000$ (only the probability at even $m$ is shown); (b) $\epsilon=3$, where the averaging has been performed over 100 realizations in the time-interval $\tau=500-10500$. Other parameters are: $h=0.2, \delta=0, N=2$. The boundaries of the resonance cells are marked by arrows. 
In Fig. 6 the classical phase space is shown for $\epsilon=3$. One can see that in the first two cells the motion is mainly chaotic while in other cells the motion remains mainly regular. The quantum probability distribution is illustrated in Fig. 7 (a) for the case of small $\epsilon$ and in Fig. 7 (b) for the case $\epsilon=3$. The initial state was taken in the form $c_{m}(0)=\delta_{m, m_{0}}$ with $m_{0}=30$ (in the center of the first cell in Figs. 7 (a), (b)). As follows from Fig. 7 (a), the quantum particle can tunnel (see also Ref.0) from the initial (first) cell to other resonance cells unlike the classical case, where practically all the trajectories in the phase space are confined inside the resonant cells (see Fig. 5 (a)).
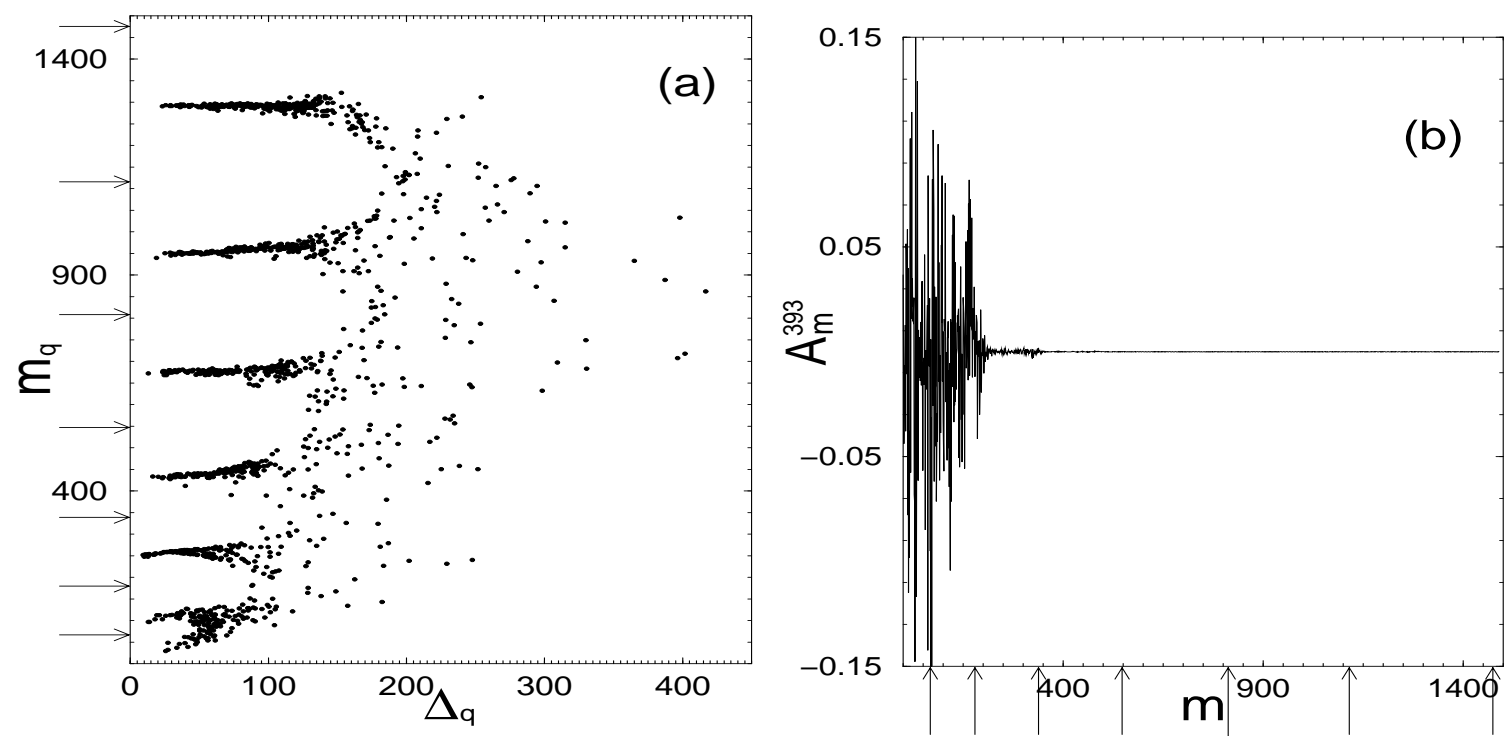

FIG. 8. (a) The plot $m_{q}$ versus $\sigma_{q}$ and (b) the characteristic QE eigenfunction located in the chaotic region for $\epsilon=3$ and $h=0.2, \delta=0, N=2$. The boundaries of the resonance cells are marked by arrows.

When $\epsilon$ increases, the probability distribution in Fig. 7 (b) increases in most of the quantum cells, which corresponds to chaotization of motion in the classical phase space. In Fig. 8 (a) we show the plot $m_{q}\left(\sigma_{q}\right)$ for the case $\epsilon=3$, and in Fig. 8 (b) the characteristic QE function located in the chaotic area is illustrated. As one can see from Fig. 8 (a), almost all QE states in the area of the first two cells are delocalized over both the cells. In other words, the QE states are localized inside the chaotic area (first two cells), but not inside the single cells, as in the case of small $\epsilon$ in Fig. 4 (a). In the chaotic regime, one can find a 
quantum particle with equal probability in any unperturbed state, $m$, inside the chaotic sea, independently of the position and the form of an initial state located in this region (see first two cells in Fig. 7 (b) and 8 (a), (b)). When $\epsilon$ increases, more classical and quantum cells become chaotic. This results in increasing the area of delocalization of the quantum chaotic states. Thus, in the regime of chaos the localization length in the SSS may be identified with the size of the chaotic area in the TPS.

\section{CONCLUSION}

The resonance approximation, given by Eqs. (21) - (24) can be interpreted in the following way. We can re-write the Hamiltonian matrix in Eq. (18) in the form,

$$
H_{m, l ; m+n, l^{\prime}}=H_{m, l ; m+n, l^{\prime}}^{0} \delta_{n, 0} \delta_{l, l^{\prime}}+F_{n, n+m} \delta_{l^{\prime}, l+1}+F_{n, n+m}^{*} \delta_{l^{\prime}, l-1} .
$$

The unperturbed motion, given by the zeroth order Hamiltonian, $H_{n, l}^{0}=h(n-\mu l)$, takes place on the infinite 1D energy surface (line) in the 2D space. The character of the motion on the energy surface depends on the form of the matrix elements of the interaction potential, $\left\langle m, l|V(X, \tau)| m^{\prime}, l^{\prime}\right\rangle=F_{m, l ; m^{\prime} l^{\prime}}$. If $V(X, \tau)$, is linear in $X$, for example, $V(X, \tau)=\epsilon X \cos \mu \tau$, and $\mu=N$, where $N=1,2, \ldots$, then the motion is unlimited 2 , 13 . When the interaction potential is nonlinear in $X, V(X, \tau)=\epsilon \cos (X-\mu \tau)$ at $\mu=N$, or when the period of the interaction potential, $\mu$, is not equal to $N \times$ (a distance between the sites of the $2 \mathrm{D}$ cell), i.e. when $\delta \neq 0$, then, as demonstrated in this paper (see also Ref. are localized.

It is necessary to note that, as was shown in 11 , the quantum and classical dynamics in the chaotic regime is practically independent of the detuning, $\delta$, when $\epsilon \gg \delta$. So, the results concerning the chaotic dynamics also remain valid in the near-resonance case.

In summary, the regular and chaotic classical and quantum dynamical regimes are analyzed in the system of an ion trapped in a linear ion trap and interacting with two laser

field with close frequencies. This system is modeled using a quantum oscillator perturbed 
by a monochromatic wave (MPO). It is shown that the problem of dynamical stability in this system corresponds to the problem of electron localization in a $2 \mathrm{D}$ solid-state system (SSS). The resonance approximation is used to decrease the effective dimensionality of the corresponding solid-state system. This can be done in the case of relatively small inter-

action between the trapped ion and the laser fields. In the SSS this case corresponds to weak interaction between the sites of the 2D lattice. Increasing the interaction amplitude results in delocalization of the quantum states over the sites of the $2 \mathrm{D}$ cell. The area of the delocalization in the SSS at strong interaction in the chaotic area may be identified with the size of the chaotic sea in the MPO. Our results provide understanding of the mechanism of stability of an ion trapped in a linear ion trap. They also allow one to estimate the characteristic dynamical regimes of the trapped ion and to choose parameters required for dynamical stability.

\section{ACKNOWLEDGMENTS}

We thank G. Chapline for useful discussions. The paper was supported by the Department of Energy under contract W-7405-ENG-36 and by the National Security Agency.

${ }^{1}$ F. Haake, Quantum Signature of Chaos (Springer-Verlag, 1991).

${ }^{2}$ L.E. Reichl, The Transition to Chaos (Springer-Verlag, New York, 1992).

${ }^{3}$ G.P. Berman, D.F.V. James, R.J. Hughes, M. S. Gulley, M.H. Holzscheiter, and G.V. López, Phys. Rev. A61, 023403 (2000).

${ }^{4}$ I.S. Gradshteyn and I.M. Ryzhik, Table of Integrals, Series, and Products, (Academic Press, New York, 1980).

${ }^{5}$ V.Ya. Demikhovskii, D.I. Kamenev, and G.A. Luna-Acosta, Phys. Rev. E59, 294 (1999). 
${ }^{6}$ M. Kohmoto and Y. Hatsugai, Phys. Rev. B41, 9527 (1990).

${ }^{7}$ V.Ya. Demikhovskii and D.I. Kamenev, Phys. Lett. A228, 391 (1997).

${ }^{8}$ V.Ya. Demikhovskii, D.I. Kamenev, and G.A. Luna-Acosta, Phys. Rev. E52, 3351 (1995).

${ }^{9}$ G.P. Berman, V.Ya. Demikhovskii, and D.I. Kamenev, Chaos 10, 670 (2000).

${ }^{10}$ G.M. Zaslavskii, R.Z. Sagdeev, D.A. Usikov, and A.A. Chernikov, Weak Chaos and Quasiregular Patterns (Cambridge University Press, Cambridge, 1991).

${ }^{11}$ G.P. Berman, D.F.V. James, and D.I. Kamenev, CHAOS 10, 371 (2000)

12 R.P.Feynman, Phys. Rev. 84, 108 (1951)

13 J. Schwinger, Phys. Rev. 91, 728 (1953) 